\title{
Comparison of clinical outcomes between in vitro fertilization (IVF) and intracytoplasmic sperm injection (ICSI) in IVF-ICSI split insemination cycles
}

\author{
Sun Hee Lee ${ }^{1,2 *}$, Jae Hyun Lee ${ }^{1 *}$, Yong-Seog Park' ${ }^{1}$ Kwang Moon Yang ${ }^{3}$, Chun Kyu Lim ${ }^{1}$ \\ 'Laboratory of Reproductive Medicine, Cheil General Hospital and Women's Healthcare Center, Dankook University College of Medicine, Seoul; \\ ${ }^{2}$ Department of Biosciences, Institute of Basic Sciences, College of Natural Sciences, Sungshin Women's University, Seoul; ${ }^{3}$ Department of Obstetrics \\ and Gynecology, Cheil General Hospital and Women's Healthcare Center, Dankook University College of Medicine, Seoul, Korea
}

Objective: This study aimed to compare the clinical outcomes between in vitro fertilization (IVF) and intracytoplasmic sperm injection (ICSI) in sibling oocytes. Additionally, we evaluated whether the implementation of split insemination contributed to an increase in the number of ICSI procedures.

Methods: A total of 571 cycles in 555 couples undergoing split insemination cycles were included in this study. Among them, 512 cycles (89.7\%) were a couple's first IVF cycle. The patients were under 40 years of age and at least 10 oocytes were retrieved in all cycles. Sibling oocytes were randomly allocated to IVF or ICSI.

Results: Total fertilization failure was significantly more common in IVF cycles than in ICSI cycles (4.0\% vs. 1.4\%, $p<0.05)$, but the low fertilization rate among retrieved oocytes (as defined by fertilization rates greater than $0 \%$ but $<30 \%$ ) was significantly higher in ICSI cycles than in IVF cycles $(17.2 \%$ vs. $11.4 \%, p<0.05)$. The fertilization rate of ICSI among injected oocytes was significantly higher than for IVF $(72.3 \% \pm 24.3 \%$ vs. $59.2 \% \pm 25.9 \%, p<0.001)$, but the fertilization rate among retrieved oocytes was significantly higher in IVF than in ICSI $(59.2 \% \pm 25.9 \%$ vs. $52.1 \% \pm 22.5 \%, p<0.001$ ). Embryo quality before embryo transfer was not different between IVF and ICSI. Although the sperm parameters were not different between the first cycle and the second cycle, split insemination or ICSI was performed in 18 of the 95 cycles in which a second IVF cycle was performed.

Conclusion: The clinical outcomes did not differ between IVF and ICSI in split insemination cycles. Split insemination can decrease the risk of total fertilization failure. However, unnecessary ICSI is carried out in most split insemination cycles and the use of split insemination might make ICSI more common.

Keywords: Fertilization failure; In vitro fertilization; Intracytoplasmic sperm injection; Low fertilization; Split insemination

\section{Introduction}

Fertilization failure can be an extremely stressful experience to cou-

Received: Nov 25, 2016 · Revised: Jan 19, 2017· Accepted: Apr 25, 2017

Corresponding author: Chun Kyu Lim

Laboratory of Reproductive Medicine, Cheil General Hospital and Women's

Healthcare Center, Dankook University College of Medicine, 17 Seoae-ro 1-gil, Jung-gu, Seoul 04619, Korea

Tel: +82-2-2000-7590 Fax:+82-2-2265-5621 E-mail: seungzzang@paran.com

*These authors contributed equally to this study.

This is an Open Access article distributed under the terms of the Creative Commons Attribution Non-Commercial License (http://creativecommons.org/licenses/by-nc/4.0/) which permits unrestricted non-commercial use, distribution, and reproduction in any medium, provided the original work is properly cited. ples undergoing in vitro fertilization (IVF) and embryo transfer (ET) cycles, as well as to clinicians. Fertilization failure occurs in $3.52 \%$ to $20 \%$ of IVF cycles [1,2], and even in $1 \%$ to $3 \%$ of intracytoplasmic sperm injection (ICSI) cycles [3,4]. Embryo transfer is usually cancelled when fertilization failure has occurred. In ICSI cycles, rescue ICSI can be performed when immature oocytes are matured 1 day after oocyte retrieval. Recently, concern over fertilization failure may have caused ICSI to become more common in cases of non-male factor infertility, especially in cycles in which a small number of oocytes are retrieved, as in patients who are poor responders or exhibit ovarian failure. Among cases of non-male factor infertility, the indications for ICSI generally include unexplained infertility, the retrieval of poor 
quality oocytes or a small number of oocytes, advanced maternal age, previous fertilization failure with conventional insemination, preimplantation genetic diagnosis (PGD), fertilization after in vitro maturation, and fertilization of frozen-thawed oocytes [5]. The main reason that ICSI is applied in such cases is to prevent fertilization failure. According to a study of the practice committees of the American Society for Reproductive Medicine and the Society for Assisted Reproductive Technology, ICSI is safe and effective for patients with male factor infertility and ICSI might benefit patients undergoing IVF cycles with PGD, in vitro matured oocytes, and frozen-thawed oocytes. However, ICSI cannot improve the clinical outcomes of patients with unexplained infertility, low oocyte yield, or old age, and there is no evidence that ICSI improves clinical outcomes in cases of nonmale factor infertility $[6,7]$. Moreover, it is unknown whether the reproductive risks known to be associated with ICSI in male factor infertility, such as increase of sex or autosomal chromosome aberrations, congenital anomalies, and imprinting disorders, are associated with ICSI in cases of non-male factor infertility [8]. The routine use of ICSI for preventing fertilization failure may decrease the incidence of fertilization failure, but ICSI must be carried out unnecessarily in more than 30 couples to prevent fertilization failure in one couple [5].

Despite the development of assisted reproductive technologies, the prediction of fertilization failure or low fertilization with perfect accuracy is impossible in IVF cycles in patients seeking treatment at infertility clinics. Fertilization failure occurs unexpectedly after conventional insemination, even though the male partner may have normal sperm parameters. ICSI is usually suggested in subsequent cycles when low fertilization or fertilization failure has occurred, although low fertilization and fertilization failure do not always occur repeatedly $[9,10]$. Additionally, ICSI is usually performed in every subsequent cycle of the couple after having been performed once. It is not easy to choose between conventional IVF and ICSI to fertilize the retrieved oocytes because there is the possibility of fertilization failure in conventional IVF, while for ICSI, there is the possibility of unnecessary implementation. Therefore, the implementation of split insemination has increased recently to minimize fertilization failure and check for the possibility of fertilization by IVF, especially in the first IVF cycle of couples with unexplained infertility [11]. Sibling oocytes are randomly allocated to conventional IVF or ICSI in split insemination. The results of fertilization after split insemination can then be helpful for determining the insemination method (IVF or ICSI) in subsequent cycles. Split insemination has mainly been implemented in patients with unexplained infertility or mild male factor infertility, and the clinical outcomes of IVF and ICSI have been compared in split insemination cycles [12-19]. However, the effectiveness of split insemination remains controversial. Fertilization failure was prevented in some IVF cycles by ICSI, but the pregnancy outcomes were not improved. Split insemination did not significantly reduce the implementation of ICSI in subsequent IVF cycles [14]. Moreover, concerns have been raised about the safety of ICSI in non-male factor infertility [5].

Recently, the application of ICSI has been greatly extended into the treatment of non-male factor infertility, although its effectiveness has not been proven in such cases. Moreover, major concerns have been raised over the implementation of unnecessary ICSI in the treatment of non-male factor infertility. The main reason for ICSI application in non-male factor infertility is the fear of fertilization failure or low fertilization. It is difficult to compare the effect of the insemination method (IVF or ICSI) on the fertilization of oocytes in separate IVF or ICSI cycles because differences among infertile couples might influence the fertilization of oocytes. Therefore, we compared the effect of the insemination method (IVF or ICSI) on fertilization in split insemination cycles to minimize the impact of such differences. We assessed the maturation status of unfertilized oocytes among inseminated oocytes to analyze whether other factors may have influenced the fertilization of oocytes. Moreover, we evaluated whether split insemination contributed to making ICSI implementation more common by analyzing the rate of ICSI implementation in the second cycles of couples in whom pregnancy was not established in their first cycle.

\section{Methods}

\section{Patients, controlled ovarian hyperstimulation, and fertilization}

In total, 555 couples underwent 571 IVF-ICSI split insemination cycles between January 2011 and December 2013. Among them, the IVF cycles of 512 couples were their first cycle. The couples included in this study had mild male factor infertility (mild asthenozoospermia, 200 cycles), tubal factor infertility (112 cycles), or unexplained infertility (259 cycles). Females aged 40 and older were excluded from this study, and only cycles in which 10 or more oocytes were retrieved were analyzed in this study. Freshly ejaculated sperm was used for fertilization. Patients with less than $50 \%$ motility in the results of a semen analysis were included in the category of mild infertility. In several cycles, there were differences in sperm concentration and motility between the semen collected on the day of oocyte pickup and the semen collected on the day of semen analysis. The clinicians explained to the patients that half of the retrieved oocytes would be fertilized by conventional IVF and the other half would be fertilized by ICSI. All couples provided informed consent.

Controlled ovarian hyperstimulation was carried out using a gonadotropin-releasing hormone antagonist, recombinant folliclestimulating hormone or human menopausal gonadotropin, and human chorionic gonadotropin (hCG). Oocytes were retrieved trans- 
vaginally under ultrasound guidance 35 hours after hCG injection. After the completion of oocyte retrieval, the retrieved cumulus-oocyte complexes were randomly allocated to receive either conventional IVF or ICSI. The oocytes allocated to IVF were maintained in fertilization medium until the sperm samples were prepared. The oocytes allocated to ICSI were prepared for ICSI. Cumulus cells were removed approximately 2 hours after oocyte retrieval. Cumulus cells were removed after short exposure to a medium containing $80 \mathrm{IU} /$ $\mathrm{mL}$ of hyaluronidase (Sage, Trumbull, CT, USA). The remaining cells were mechanically removed by gentle pipetting with hand-drawn glass capillaries. The maturation status of the cumulus cell-removed oocytes was evaluated using an inverted microscope (Nikon, Tokyo, Japan). The oocytes were maintained in fertilization medium for at least 1 hour before ICSI. Semen samples were collected immediately after oocyte pick-up. Sperm concentration and motility were analyzed by light microscopy. Sperm was prepared by the density gradient centrifugation method using SpermGrad (Vitrolife, Göteborg, Sweden). The oocytes allocated to IVF were inseminated with sperm within 5 hours after oocyte retrieval. The oocytes were placed in fertilization medium with 50,000 or more motile spermatozoa per milliliter overnight. Among the oocytes allocated to ICSI, mature metaphase Il oocytes were injected with sperm.

\section{Assessment of fertilization and embryo quality}

Fertilization was assessed approximately 16 hours after insemination or ICSI. Normal fertilization was confirmed by the presence of 2 pronuclei (PN) and the extrusion of the second polar body (PB). In all split insemination cycles, the fertilization rate of IVF and the fertilization rate of ICSI were calculated independently. The fertilization rate of conventional IVF was calculated as the percentage of fertilized oocytes of the inseminated (or retrieved) oocytes. In ICSI, two fertilization rates were calculated: the percentage of fertilized oocytes among the injected oocytes and the percentage of fertilized oocytes among the retrieved oocytes (oocytes that were allocated to ICSI). After the fertilization status of the oocytes was observed, fertilized oocytes were cultured in a 50- $\mu \mathrm{L}$ drop of medium covered with paraffin oil, in a humidified atmosphere under $6 \% \mathrm{CO}_{2}$ at $37^{\circ} \mathrm{C}$ before embryo transfer. Embryos fertilized by conventional IVF and embryos fertilized by ICSI were cultured separately. Embryos were replaced between day 2 and day 5. Embryo quality was assessed just before embryo transfer. Embryo quality was classified according to the embryo morphology on the day of embryo transfer. Comparison of embryo quality between conventional IVF and ICSI was performed only in cycles in which embryo transfer was performed on day 3 after oocyte retrieval. Day 3 embryo transfers were performed in 301 cycles. Embryos were classified as good, fair, or poor embryos based on their morphology. Good embryos had $\geq 7$ even-sized blastomeres and $\leq 10 \%$ fragmentation of the volume of the embryos. Fair embryos had $\leq 6$ even-sized or $\geq 6$ uneven-sized blastomeres and $10 \%$ to $30 \%$ fragmentation. Poor embryos had uneven-sized blastomeres, regardless of the blastomere number, and $>30 \%$ fragmentation. Embryos from conventional IVF were replaced in 166 cycles; embryos from ICSI were replaced in 66 cycles; and embryos from conventional IVF were replaced along with embryos from ICSI in 278 cycles. Clinical pregnancy was ascertained by confirming the fetal heart beat using ultrasonography at 6 or 7 weeks of gestation.

\section{Statistical analysis}

Results are expressed as mean \pm standard deviation. Statistical analysis was performed using SPSS ver. 12.0 for Windows (SPSS Inc., Chicago, IL, USA). One-way analysis of variance, the Kruskal-Wallis rank test, or the Fisher exact test was performed to analyze the significance of differences between groups. A $p$-value of $<0.05$ was considered to indicate a statistically significant difference.

\section{Results}

\section{Comparison of fertilization rates between conventional IVF and ICSI in IVF-ICSI split insemination cycles}

A total of 10,471 oocytes were retrieved in 571 cycles. Among them, 4,915 oocytes were allocated to IVF and 5,556 oocytes were allocated to ICSI. In IVF, normal fertilization (2 PN and second PB) was observed in 2,878 oocytes (58.6\%). In ICSI, 3,926 oocytes were injected with sperm, and normal fertilization was observed in 2,854 oocytes (51.4\%). The fertilization rates of conventional IVF and ICSI were compared in IVF-ICSI split insemination cycles (Table 1). The fertilization rate among the injected oocytes in the ICSI group (72.3\% $\pm 24.3 \%)$ was significantly higher $(p<0.001)$ than the fertilization rate among the inseminated (or retrieved) oocytes after conventional IVF $(59.2 \% \pm$ $25.9 \%)$. However, fertilization rate among the retrieved oocytes in the ICSI group $(52.1 \% \pm 22.5 \%)$ was significantly lower $(p<0.001)$ than the fertilization rate among the inseminated (or retrieved) oocytes after conventional IVF $(59.2 \% \pm 25.9 \%)$.

The occurrence of total fertilization failure and low fertilization (fertilization rate $<30 \%$ ) were compared between conventional IVF and ICSI in split insemination cycles (Table 2). Total fertilization failure occurred in 23 cycles of conventional IVF (4.0\%) and in 8 cycles of ICSI (1.4\%) among the 571 cycles. Total fertilization failure occurred significantly more often $(p<0.05)$ in conventional IVF than in ICSI. The low fertilization rate among the inseminated oocytes in the conventional IVF group was $11.4 \%$ (65 of 571) and the low fertilization rate among the injected oocytes in the ICSI group was 7.0\% (32 of 571). The low fertilization rate was significantly higher $(p<0.05)$ in the conventional IVF group than in the ICSI group when the fertilization 
Table 1. Comparison of fertilization rate between IVF and ICSI in split insemination cycles

\begin{tabular}{|c|c|c|c|c|}
\hline Variable & Total & Mild male factor & Tubal factor & Unexplained factor \\
\hline No. of cycles & 571 & 200 & 112 & 259 \\
\hline No. of retrieved oocytes & $18.3 \pm 7.9$ & $18.4 \pm 7.6$ & $18.5 \pm 7.0$ & $18.2 \pm 7.8$ \\
\hline Fertilization rate of ICSI (among injected oocytes) (\%) & $72.3 \pm 24.3^{b)}$ & $72.5 \pm 23.9^{b)}$ & $72.2 \pm 25.1^{\mathrm{b})}$ & $72.1 \pm 24.4^{b)}$ \\
\hline Fertilization rate of ICSI (among retrieved oocytes) (\%) & $52.1 \pm 22.5^{c)}$ & $53.6 \pm 22.6$ & $53.6 \pm 23.0$ & $50.3 \pm 22.1^{c)}$ \\
\hline No. of embryo transfer cycles & 510 & 183 & 98 & 229 \\
\hline No. of cycles with pregnancy (\%) & $243(47.6)$ & 89 (48.6) & $51(52.0)$ & $103(45.0)$ \\
\hline
\end{tabular}

Values are presented as mean \pm standard deviation unless otherwise indicated.

IVF, in vitro fertilization; ICSI, intracytoplasmic sperm injection.

a) vs.b); a) vs.c) Significantly different from each other $(p<0.001)$.

Table 2. Comparison of total fertilization failure and low fertilization between IVF and ICSI in split insemination cycles

\begin{tabular}{|c|c|c|c|c|c|c|}
\hline \multirow[b]{2}{*}{ Cause of infertility } & \multirow[b]{2}{*}{ No. of cycles } & \multicolumn{2}{|c|}{ IVF } & \multicolumn{3}{|c|}{ ICSI } \\
\hline & & $\begin{array}{l}\text { Total fertilization } \\
\text { failure }\end{array}$ & $\begin{array}{c}\text { Low fertilization } \\
\text { (among retrieved } \\
\text { oocytes) }\end{array}$ & $\begin{array}{l}\text { Total fertilization } \\
\text { failure }\end{array}$ & $\begin{array}{l}\text { Low fertilization } \\
\text { (among injected } \\
\text { oocytes) }\end{array}$ & $\begin{array}{c}\text { Low fertilization } \\
\text { (among retrieved } \\
\text { oocytes) }\end{array}$ \\
\hline Total & 571 & $23(4.0)^{a)}$ & $65(11.4)^{b)}$ & $8(1.4)^{c)}$ & $32(7.0)^{d)}$ & $90(17.2)^{\mathrm{e})}$ \\
\hline Mild male factor & 200 & $10(5.0)^{\mathrm{a})}$ & $21(10.5)$ & $2(1.0)^{c)}$ & $11(5.5)$ & $30(15.0)$ \\
\hline Tubal factor & 112 & $5(4.5)$ & $14(12.5)$ & $3(2.7)$ & $6(5.4)$ & $14(12.5)$ \\
\hline
\end{tabular}

Values are presented as number (\%).

IVF, in vitro fertilization; ICSI, intracytoplasmic sperm injection.

a) vs. c); b vs. d); b vs.e) Significantly different from each other $(p<0.05)$.

Table 3. Comparison of patient characteristics among couples with different infertility factors

\begin{tabular}{|c|c|c|c|c|c|}
\hline Variable & Total & Mild male factor & Tubal factor & Unexplained factor & $p$-value \\
\hline No. of cycles & 571 & 200 & 112 & 259 & - \\
\hline Female age (yr) & $34.0 \pm 2.9$ & $34.1 \pm 2.8^{\mathrm{a})}$ & $33.0 \pm 3.3^{b)}$ & $34.3 \pm 2.8^{\mathrm{a})}$ & $<0.01$ \\
\hline FSH level (mlU/mL) & $6.7 \pm 1.9$ & $6.6 \pm 1.8$ & $6.4 \pm 2.0$ & $6.9 \pm 1.9$ & 0.075 \\
\hline Estradiol level (pg/mL) & $3,670.6 \pm 1,748.2$ & $3,876.1 \pm 1,990.2$ & $3,921.9 \pm 1,947.2$ & $3,533.9 \pm 1,588.0$ & 0.334 \\
\hline Sperm concentration before treatment $\left(\times 10^{6} / \mathrm{mL}\right)$ & $108.8 \pm 66.5$ & $104.5 \pm 72.9$ & $96.4 \pm 56.2^{c)}$ & $117.6 \pm 30.5^{d)}$ & $<0.01$ \\
\hline Sperm motility before treatment (\%) & $57.3 \pm 26.7$ & $41.4 \pm 20.2^{\mathrm{e})}$ & $61.1 \pm 17.0^{f)}$ & $50.9 \pm 11.7^{f)}$ & $<0.01$ \\
\hline Sperm concentration after treatment $\left(\times 10^{6} / \mathrm{mL}\right)$ & $29.4 \pm 11.9$ & $26.1 \pm 12.1^{\mathrm{e})}$ & $29.5 \pm 11.9$ & $32.0 \pm 11.2^{f)}$ & $<0.01$ \\
\hline Sperm motility after treatment (\%) & $90.8 \pm 8.6$ & $88.0 \pm 10.7^{e)}$ & $92.6 \pm 5.1^{f)}$ & $92.1 \pm 7.3^{f)}$ & $<0.01$ \\
\hline
\end{tabular}

Values are presented as mean \pm standard deviation.

$\mathrm{FSH}$, follicle-stimulating hormone.

Significantly different from each other in the same column: ${ }^{\text {a) vs. bl }} p<0.01$; ${ }^{\text {(c) vs d) }} p<0.05$; ${ }^{\text {e) vs.ff }} p<0.01$.

rate was calculated among the inseminated oocytes in IVF and among the injected oocytes in ICSI. However, the low fertilization rate among retrieved oocytes in the conventional IVF group (11.4\%) was significantly lower $(p<0.05)$ than the corresponding rate in the ICSI group (17.2\%).

\section{Comparison of fertilization rates between conventional IVF and ICSI in couples with different infertility factors}

The fertilization rates of conventional IVF and ICSI were compared in couples with three different infertility factors: mild asthenozoospermia, tubal factor, and unexplained factor. The female partners were significantly younger $(p<0.001)$ in couples with tubal factor infertility $(33.0 \pm 3.3$ years) than in couples with mild male factor or unexplained factor infertility ( $34.1 \pm 2.8$ years and $34.3 \pm 2.8$ years, respectively). The number of retrieved oocytes, the follicle-stimulating hormone level on day 2 or 3 of the menstrual cycle, and the estradiol level on the day before oocyte retrieval were not different among couples with the various infertility factors (Table 3). The clinical preg- 
Table 4. Comparison of embryo quality between IVF and ICSI in split insemination cycles

\begin{tabular}{|c|c|c|c|c|c|c|c|}
\hline \multirow{3}{*}{ Cause of infertility } & \multirow{3}{*}{ No. of cycles } & \multicolumn{6}{|c|}{ Embryo quality (\%) } \\
\hline & & \multicolumn{3}{|c|}{ IVF } & \multicolumn{3}{|c|}{ ICSI } \\
\hline & & Good & Fair & Poor & Good & Fair & Poor \\
\hline Total & 301 & $16.6 \pm 23.2$ & $47.7 \pm 29.4^{\text {a) }}$ & $35.6 \pm 29.6^{b)}$ & $16.6 \pm 26.6$ & $53.0 \pm 34.8^{c}$ & $29.8 \pm 33.4^{d}$ \\
\hline Mild male factor & 108 & $16.9 \pm 22.2$ & $47.9 \pm 30.5$ & $35.1 \pm 29.8$ & $17.8 \pm 26.3$ & $53.5 \pm 34.3$ & $28.9 \pm 33.0$ \\
\hline Tubal factor & 59 & $21.0 \pm 28.1$ & $48.1 \pm 30.7$ & $30.9 \pm 29.3$ & $16.3 \pm 25.3$ & $54.4 \pm 34.8$ & $27.2 \pm 31.8$ \\
\hline Unexplained factor & 134 & $14.5 \pm 21.5$ & $47.4 \pm 28.1$ & $38.0 \pm 29.6$ & $15.8 \pm 27.6$ & $52.1 \pm 35.5$ & $31.6 \pm 34.6$ \\
\hline
\end{tabular}

Values are presented as mean \pm standard deviation.

IVF, in vitro fertilization; ICSI, intracytoplasmic sperm injection.

a) vs.c); b) vs. d) Significantly different from each other $(p<0.05)$.

nancy rate was not different among these couples (Table 1). Sperm concentration and motility before and after preparation for IVF were compared (Table 3). Differences were found in sperm concentration and motility before the preparation of sperm for IVF among these couples. The sperm concentration of couples with tubal factor infertility $\left(95.4 \pm 56.5 \times 10^{6} / \mathrm{mL}\right)$ was significantly lower $(p<0.05)$ than that of couples with unexplained factor infertility $\left(117.6 \pm 30.5 \times 10^{6} \%\right.$ $\mathrm{mL}$ ). The sperm concentration of couples with mild asthenozoospermia $\left(104.5 \pm 72.9 \times 10^{6} / \mathrm{mL}\right)$ was not different compared to those of the couples with other infertility factors. The sperm motility of couples with mild asthenozoospermia $(41.4 \% \pm 20.2 \%)$ was significantly lower $(p<0.01)$ than was observed in couples with tubal factor or unexplained factor infertility $(61.1 \% \pm 17.0 \%$ and $50.95 \% \pm 11.7 \%$, respectively). There were differences in sperm concentration and motility after sperm preparation for IVF using the discontinuous density gradient method among the couples with these three different infertility factors. The sperm concentration of couples with mild asthenozoospermia $\left(26.1 \pm 12.1 \times 10^{6} / \mathrm{mL}\right)$ was significantly lower $(p<0.01)$ than that of couples with unexplained factor infertility $\left(32.0 \pm 11.2 \times 10^{6} / \mathrm{mL}\right)$. The sperm concentration of couples with tubal factor infertility $\left(29.5 \pm 11.9 \times 10^{6} / \mathrm{mL}\right)$ was not significantly different than that observed in couples with mild asthenozoospermia or unexplained factor infertility. The sperm motility of couples with mild asthenozoospermia $(88.0 \% \pm 10.7 \%)$ was significantly lower $(p<$ 0.01 ) than in couples with tubal factor or unexplained factor infertility $(92.6 \% \pm 5.4 \%$ and $92.1 \% \pm 7.3 \%$, respectively).

The fertilization rate among the injected oocytes in the ICSI group was significantly higher $(p<0.001)$ than the fertilization rate among the inseminated oocytes in the conventional IVF group in all couples with all three different infertility factors. However, the fertilization rate among the retrieved oocytes in the ICSI group was lower than the fertilization rate among the inseminated oocytes that underwent conventional IVF (Table 1). In couples with mild asthenozoospermia, total fertilization failure was significantly more common $(p<0.05)$ in the conventional IVF group (5.0\%) than in the ICSI group (1.0\%), while the occurrence of low fertilization was not different between conventional IVF and ICSI. In couple with tubal factor infertility, the total fertilization and low fertilization rates were not different between conventional IVF and ICSI. In couples with unexplained factor infertility, the occurrence of total fertilization failure was not different between conventional IVF and ICSI, but the low fertilization rate among the inseminated oocytes in the conventional IVF group $(11.6 \%)$ was significantly higher $(p<0.05)$ than the low fertilization rate among the injected oocytes in the ICSI group (5.8\%) (Table 2).

\section{Comparison of embryo quality between conventional IVF and ICSI in split insemination cycles and pregnancy outcomes depending on the origin of the transferred embryos}

The quality of the embryos fertilized by conventional IVF or ICSI was compared in the 301 cycles in which embryos were transferred on day 3 (Table 4). The rate of good-quality embryos was not different between embryos from conventional IVF $(16.6 \% \pm 23.2 \%)$ and embryos from ICSI $(16.6 \% \pm 26.6 \%)$. Fair-quality embryos were significantly more common $(p<0.05)$ in embryos from ICSI $(53.0 \% \pm 34.8 \%)$ than in embryos from conventional IVF $(47.7 \% \pm 29.4 \%)$, and poorquality embryos were significantly more common $(p<0.05)$ in embryos from conventional IVF $(35.6 \% \pm 29.6 \%)$ than in embryos from ICSI (29.8\% $\pm 33.4 \%)$.

All embryos were frozen in 59 cycles, embryo transfer was cancelled due poor embryo quality in 2 cycles, and embryos were replaced in 510 cycles. Positive $\beta$-hCG results were observed in 276 cycles and 31 cycles ended in biochemical pregnancies. Of the 245 clinical pregnancies, eight were ectopic pregnancies; 21 were aborted within the first trimester of gestation; four were terminated; four pregnancies were lost in the second trimester of gestation; and 168 pregnancies resulted in live births. The main cause of termination was incompetent internal os of cervix. Follow-up was not carried out for 40 pregnancies because contact with the patients was lost after the clinical pregnancy was confirmed. Pregnancy outcomes were analyzed according to the origin of the transferred embryos (Table 5). 
Table 5. Pregnancy outcomes according to the origin of transferred embryos in split insemination cycles

\begin{tabular}{|c|c|c|c|c|c|}
\hline \multirow{2}{*}{ Variable } & \multicolumn{4}{|c|}{ Origin of transferred embryos } & \multirow{2}{*}{$p$-value } \\
\hline & IVF/ICSI & ICSI & IVF & Total & \\
\hline No. of ET cycles & 278 & 66 & 166 & 510 & - \\
\hline Female age (yr) & $34.4 \pm 3.1^{\mathrm{a})}$ & $33.8 \pm 2.8$ & $33.6 \pm 2.6^{b)}$ & $34.1 \pm 2.9$ & 0.033 \\
\hline No. of cultured embryos & $7.9 \pm 2.2^{c)}$ & $7.3 \pm 2.9$ & $8.5 \pm 2.4^{\mathrm{d})}$ & $8.0 \pm 2.4$ & 0.001 \\
\hline No. of transferred embryos & $3.3 \pm 0.7^{e)}$ & $2.8 \pm 0.8^{f)}$ & $2.8 \pm 0.2^{f)}$ & $3.1 \pm 0.8$ & $<0.001$ \\
\hline$(+) \beta-h\left(G^{g}\right)$ & $155(55.8)$ & $35(53)$ & $91(54.8)$ & $276(54.1)$ & $0.920^{\mathrm{h})}$ \\
\hline Clinical pregnancy ${ }^{\mathrm{g}}$ & $132(47.5)$ & $33(50.0)$ & $80(48.2)$ & $245(48.0)$ & $0.933^{\mathrm{h})}$ \\
\hline Ectopic pregnancy ${ }^{i)}$ & $4(3.0)$ & - & $4(5.0)$ & $8(3.3)$ & $0.387^{j)}$ \\
\hline Clinical abortion & $10(7.6)$ & $3(9.1)$ & $8(10.0)$ & $21(8.6)$ & $0.824^{i)}$ \\
\hline Second trimester lossi) & $3(2.3)$ & $1(3.0)$ & $1(1.3)$ & $4(1.6)$ & $0.620^{i)}$ \\
\hline Terminationi) & $2(1.5)$ & $1(3.0)$ & $1(1.3)$ & $4(1.6)$ & $0.784)$ \\
\hline Follow-up loss ${ }^{i)}$ & $23(17.4)$ & $3(9.1)$ & $14(17.5)$ & $40(16.3)$ & $0.481^{\mathrm{j})}$ \\
\hline Live birthi) & $90(68.5)$ & $26(78.8)$ & $52(65.0)$ & $168(68.6)$ & $0.353^{\mathrm{j})}$ \\
\hline
\end{tabular}

Values are presented as mean \pm standard deviation or number (\%).

IVF, in vitro fertilization; ICSI, intracytoplasmic sperm injection; ET, embryo transfer; $\mathrm{hCG}$, human chorionic gonadotropin.

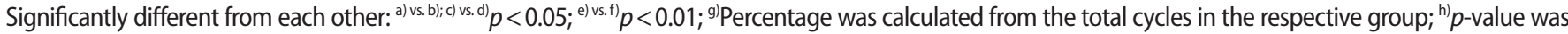
calculated from the total cycles in the respective group; i Percentage was calculated from the clinical pregnancies in the respective group; ${ }^{j} p$-value was calculated from the total clinical pregnancies in the respective group.

Table 6. Insemination methods of subsequent cycles and fertilization rate of respective insemination methods of the first cycle

\begin{tabular}{|c|c|c|c|c|c|}
\hline \multirow{2}{*}{$\begin{array}{l}\text { Insemination method of } \\
\text { subsequent cycles }\end{array}$} & \multicolumn{4}{|c|}{ Fertilization rate of the respective insemination method in the first cycle } & \multirow{2}{*}{ Total } \\
\hline & IVF $\geq 50 \%$ ICSI $\geq 50 \%$ & IVF $\geq 50 \%$ ICSI $<50 \%$ & IVF $<50 \%$ ICSI $\geq 50 \%$ & IVF $<50 \%$ ICSI $<50 \%$ & \\
\hline IVF & $20(21.1)$ & $18(18.9)$ & - & $8(8.4)$ & $46(48.4)$ \\
\hline IVF+ICSI & $7(7.4)$ & $7(7.4)$ & $8(8.4)$ & $5(5.3)$ & $27(28.4)$ \\
\hline ICSI & $3(3.2)$ & $2(2.1)$ & $10(10.5)$ & $7(7.4)$ & $22(23.2)$ \\
\hline Total & $30(31.6)$ & $27(28.4)$ & $18(18.9)$ & $20(21.1)$ & 95 \\
\hline
\end{tabular}

Values are presented as number (\%).

IVF, in vitro fertilization; ICSI, intracytoplasmic sperm injection.

Only embryos from conventional IVF were replaced in 166 cycles. Fertilization failure occurred in 4 ICSI cycles, and all fertilized oocytes from ICSI were frozen in 64 cycles. In these cycles, the mean age of the female partner was $33.6 \pm 2.6$ years old, and the mean number of replaced embryos was $2.8 \pm 0.2$. Clinical pregnancy was confirmed in 80 cycles (48.2\%). In 66 cycles, only embryos from ICSI were replaced. Fertilization failure occurred in 17 IVF cycles, and all fertilized oocytes from IVF were frozen in 1 cycle. The mean age of the female partner was $33.8 \pm 2.8$ years old and the mean number of replaced embryos was $2.8 \pm 0.8$. Clinical pregnancy was confirmed in 33 cycles (50.0\%). Embryos from conventional IVF were replaced along with embryos from ICSI in the remaining 278 cycles, and clinical pregnancy was confirmed in 132 cycles (47.5\%). The mean age of the female partner was $34.4 \pm 3.1$ years old and the mean number of replaced embryos was $3.3 \pm 0.7$ in these cycles. Female age was significantly higher $(p<0.05)$ in patients in whom embryos from IVF were transferred along with embryos from ICSI than in patients in whom only embryos from IVF were transferred. Moreover, significantly more $(p<0.05)$ embryos were cultured in patients in whom only embryos from IVF were transferred $(8.5 \pm 2.4)$ than in patients in whom embryos from IVF were transferred along with embryos from ICSI $(7.9 \pm 2.2)$ or in patients in whom only embryos from ICSI were transferred (7.3 \pm 2.9$)$. Significantly more $(p<0.001)$ embryos were transferred in patients in whom embryos from IVF were transferred along with embryos from ICSI $(3.3 \pm 0.7)$ than in patients in whom only embryos from ICSI were transferred $(2.8 \pm 0.8)$ or in patients in whom only embryos from IVF were transferred $(2.8 \pm 0.2)$. However, the rates of clinical pregnancy, abortion, ectopic pregnancy, termination, second trimester loss, and live birth were not significantly different among these three groups $(p=0.933)$.

\section{Contribution of IVF-ICSI split insemination to the increase of ICSI cycles}

IVF-ICSI split insemination was performed in 571 cycles in 555 couples. Among them, the IVF cycles of 521 couples were their first cycles. Ninety-five couples did not become pregnant in their first cycle 
and underwent a second IVF cycle. The insemination techniques used in the second cycles were analyzed: conventional IVF, ICSI, or split insemination (Table 6). The insemination method of the second cycle was analyzed depending on the fertilization rate of conventional IVF and ICSI in their first cycles. In 30 of the 95 second cycles, the fertilization rates of conventional IVF and ICSI in the first cycle were over 50\%. Among them, conventional IVF was carried out in 20 cycles, split insemination in 7 cycles, and ICSI in 3 cycles. In 27 of the second cycles, the fertilization rate of conventional IVF of the first cycle was over $50 \%$ while the fertilization rate of ICSI in the first cycle was under 50\%. In this group, conventional IVF was carried out in 18 cycles, split insemination in 7 cycles, and ICSI in 2 cycles. In 18 of the second cycles, the fertilization rate of conventional IVF in the first cycle was under $50 \%$ and the fertilization rate of ICSI in the first cycle was over $50 \%$. In the second cycles in this group, conventional IVF was not carried out, split insemination was carried out in 8 cycles, and ICSI was performed in 10 cycles. In 20 of the second cycles, both the fertilization rates of conventional IVF and ICSI in the first split insemination cycle was under $50 \%$. In this group, conventional IVF was carried out in 8 cycles, split insemination in 5 cycles, and ICSI in 7 cycles. Split insemination or ICSI was carried out in 19 cycles although the fertilization rate of conventional IVF was over $50 \%$ in the first cycle. Sperm concentration and motility $\left(93.1 \pm 41.4 \times 10^{6} / \mathrm{mL}, 56.5 \% \pm\right.$ $19.3 \%$ ) did not significantly change in the second cycle in comparison to the first cycle $\left(102.5 \pm 61.1 \times 10^{6} / \mathrm{mL}, 47.9 \% \pm 15.4 \%\right)$. ICSI should be carried out in the first cycle since sperm concentration and motility in the second cycle $\left(6.5 \times 10^{6} / \mathrm{mL}, 19.0 \%\right)$ were lower than the corresponding values observed in the first cycle $\left(24.3 \times 10^{6} / \mathrm{mL}\right.$, $32.9 \%)$.

\section{Discussion}

In split insemination cycles, the fertilization rate has been reported to be somewhat different between IVF and ICSI in multiple studies. In many studies, the fertilization rate of ICSI was reported to be higher than that of IVF when the fertilization rates were calculated based on the oocytes allocated to IVF or ICSI [19-22]. However, it has been reported that the fertilization rate of IVF was similar to or higher than that of ICSI in several studies $[6,17,23]$. In the present study, the fertilization rate was significantly higher in IVF than in ICSI when the fertilization rate was calculated based on the allocated oocytes. We observed the maturation status of the unfertilized oocytes among inseminated oocytes when their fertilization status was checked the morning after oocyte retrieval. Of the 10,471 oocytes, 4,915 were allocated to IVF. Among them, 2,878 oocytes were normally fertilized ( 2 PN and 2 PB, 58.6\%) and 392 oocytes were abnormally fertilized (1 $\mathrm{PN}$ or $\geq 3 \mathrm{PN}, 8.0 \%)$. Of the oocytes, 771 were metaphase II oocytes
(15.7\%), 749 were immature oocytes (metaphase I or germinal vesicle stage, $15.2 \%$ ) and 125 oocytes were abnormal or degenerated oocytes (2.5\%). Immature, abnormal, or degenerated oocytes cannot be fertilized. Some of the metaphase II oocytes were immature at the time of oocyte retrieval and underwent maturation between oocyte retrieval and observation of their fertilization status (about 1 day), and the others were mature at the time of oocyte retrieval but were not fertilized. In contrast, some of the fertilized oocytes were immature at the time of oocyte retrieval but underwent maturation and were fertilized between oocyte retrieval and observation of their fertilization status. According to these results, most of the oocytes that were mature at the time of oocyte retrieval were fertilized, while very few oocytes could not be fertilized. The practice committees of the American Society for Reproductive Medicine and Society for Assisted Reproductive Technology have reported that fertilization failure seems to be correlated with poor ovarian stimulation [5]. Fertilization after IVF also seems to be correlated with ovarian stimulation. Therefore, the fertilization rate after IVF seems to be improved if ovarian stimulation is carried out in a way that increases the rate of mature oocytes among the retrieved oocytes, although the fertilization of oocytes is influenced by many factors.

Similar to recent studies, fertilization failure occurred more frequently in IVF cycles than in ICSI cycles in the present study. Low fertilization, corresponding to a rate of less than $30 \%$, was also more common in IVF than ICSI when fertilization rate of ICSI was calculated based on the injected oocytes. However, low fertilization was significantly more common in the ICSI cycles than in the IVF cycles when it was calculated based on the allocated oocytes. Low fertilization depending on the infertility indication was also similar between IVF and ICSI or was higher in ICSI than in IVF when the fertilization rate was calculated based on the allocated oocytes, although the difference was not significant. Fertilization failure was significantly more common in couples with only mild male factor infertility when fertilization failure was analyzed based on the infertility indication. According to these results, it seems that ICSI is not beneficial in terms of fertilization compared with IVF. However, ICSI may decrease the incidence of fertilization failure in couples with some infertility indications, such as mild male factor infertility. But ICSI is unnecessarily implemented in most of those couples to prevent the fertilization failure in some couples of them.

The rate of good-quality embryos did not differ significantly between IVF and ICSI. This result is consistent with the results of other studies [22,24-28]. However, the fair-quality embryo rate was significantly higher in ICSI than in IVF and the poor-quality embryo rate was significantly higher in IVF than in ICSI. Overall, the quality of embryos was better in ICSI than in IVF. The reason for this is likely that some of the IVF embryos were derived from immature oocytes. In 
particular, some oocytes that were immature at the time of oocyte retrieval underwent maturation and were fertilized during IVF, and the embryos derived from theses oocytes were mixed in with the embryos derived from in vivo matured oocytes. In contrast, all ICSI embryos were derived from oocytes that underwent maturation before ICSI was implemented. For this reason, it seems that the overall quality of ICSI embryos was better than that of the IVF embryos. It is difficult to evaluate the developmental potential of embryos derived from immature oocytes matured during IVF. According to the clinical outcomes of ICSI, the developmental potential of embryos derived from in vitro matured oocytes is inferior to that of embryos derived from in vivo matured oocytes. The cleavage rate of oocytes fertilized from an in vitro matured oocyte was lower than that of fertilized oocytes that were in metaphase II at the time of denudation [29-31]. Moreover, the good-quality embryo rate was lower in embryos derived from in vitro matured oocytes than in embryos derived from mature oocytes [32]. The pregnancy outcomes did not differ among cycles depending on the origin of the replaced embryos; this finding was based on a comparison of cycles in which only IVF embryos were replaced, cycles in which only ICSI embryos were replaced, and cycles in which IVF embryos were replaced together with ICSI embryos.

There are limitations to our study. The selection of transferred embryos could not be controlled due to the retrospective design of this study. Embryos with good quality were preferentially selected for embryo transfer, and embryos from IVF were preferentially selected over the embryos from ICSI when the embryo quality was similar between them. Therefore, there are limitations in the comparison of pregnancy rates among study groups. We analyzed as many split insemination cycles as possible to overcome such limitations. We additionally limited the age of the female patients ( $<40$ years old), the number of retrieved oocytes $(\geq 10)$ and the infertility factors of the patients. However, a prospective randomized study is needed on a large scale to analyze whether split insemination is effective.

In conclusion, the fertilization rate and embryo quality were not significantly different between IVF and ICSI in split insemination cycles. Total fertilization failure was significantly less common in ICSI cycles than in IVF cycles. However, the fertilization rate of IVF was similar to or higher than that of ICSI in $78.9 \%$ of the split insemination cycles ( 451 of 571 cycles). Unnecessary ICSI was therefore implemented in these cycles. For this reason, the implementation of split insemination should be considered carefully.

\section{Conflict of interest}

No potential conflict of interest relevant to this article was reported.

\section{Acknowledgments}

The authors thank the staff members of the Laboratory of Reproductive Medicine, Cheil General Hospital and Women's Healthcare Center.

\section{References}

1. Combelles CM, Morozumi K, Yanagimachi R, Zhu L, Fox JH, Racowsky $C$. Diagnosing cellular defects in an unexplained case of total fertilization failure. Hum Reprod 2010;25:1666-71.

2. Ming L, Liu P, Qiao J, Lian Y, Zheng X, Ren X, et al. Synchronization between embryo development and endometrium is a contributing factor for rescue ICSI outcome. Reprod Biomed Online 2012;24:527-31.

3. Flaherty SP, Payne D, Matthews CD. Fertilization failures and abnormal fertilization after intracytoplasmic sperm injection. Hum Reprod 1998;13 Suppl 1:155-64.

4. Esfandiari N, Javed MH, Gotlieb L, Casper RF. Complete failed fertilization after intracytoplasmic sperm injection: analysis of 10 years' data. Int J Fertil Womens Med 2005;50:187-92.

5. Practice Committees of the American Society for Reproductive Medicine and Society for Assisted Reproductive Technology. Intracytoplasmic sperm injection (ICSI) for non-male factor infertility: a committee opinion. Fertil Steril 2012;98:1395-9.

6. Bhattacharya S, Hamilton MP, Shaaban M, Khalaf Y, Seddler M, Ghobara T, et al. Conventional in-vitro fertilisation versus intracytoplasmic sperm injection for the treatment of non-male-factor infertility: a randomised controlled trial. Lancet 2001;357:20759.

7. Check JH, Yuan W, Garberi-Levito MC, Swenson K, McMonagle K. Effect of method of oocyte fertilization on fertilization, pregnancy and implantation rates in women with unexplained infertility. Clin Exp Obstet Gynecol 2011;38:203-5.

8. Practice Committee of American Society for Reproductive Medicine. Intracytoplasmic sperm injection (ICSI). Fertil Steril 2008; 90(5 Suppl):S187.

9. van der Westerlaken L, Helmerhorst F, Dieben S, Naaktgeboren N. Intracytoplasmic sperm injection as a treatment for unexplained total fertilization failure or low fertilization after conventional in vitro fertilization. Fertil Steril 2005;83:612-7.

10. Kinzer DR, Barrett CB, Powers RD. Prognosis for clinical pregnancy and delivery after total fertilization failure during conventional in vitro fertilization or intracytoplasmic sperm injection. Fertil Steril 2008;90:284-8.

11. Quaas A, Dokras A. Diagnosis and treatment of unexplained infertility. Rev Obstet Gynecol 2008;1:69-76. 
12. Khamsi F, Yavas Y, Roberge S, Wong JC, Lacanna IC, Endman M. Intracytoplasmic sperm injection increased fertilization and good-quality embryo formation in patients with non-male factor indications for in vitro fertilization: a prospective randomized study. Fertil Steril 2001;75:342-7.

13. Hershlag A, Paine T, Kvapil G, Feng H, Napolitano B. In vitro fertilization-intracytoplasmic sperm injection split: an insemination method to prevent fertilization failure. Fertil Steril 2002;77:22932.

14. Plachot M, Belaisch-Allart J, Mayenga JM, Chouraqui A, Tesquier $\mathrm{L}$, Serkine AM. Outcome of conventional IVF and ICSI on sibling oocytes in mild male factor infertility. Hum Reprod 2002;17:3629.

15. Kihaile PE, Misumi J, Hirotsuru K, Kumasako Y, Kisanga RE, Utsunomiya T. Comparison of sibling oocyte outcomes after intracytoplasmic sperm injection and in vitro fertilization in severe teratozoospermic patients in the first cycle. Int J Androl 2003;26:5762.

16. van der Westerlaken L, Naaktgeboren N, Verburg H, Dieben S, Helmerhorst FM. Conventional in vitro fertilization versus intracytoplasmic sperm injection in patients with borderline semen: a randomized study using sibling oocytes. Fertil Steril 2006;85: 395-400.

17. Fan W, Li SW, Li L, Huang Z, Ma Q, Wang Y, et al. Outcome of conventional IVF and ICSI on sibling oocytes in the case of isolated teratozoospermia. J Assist Reprod Genet 2012;29:905-10.

18. Johnson LN, Sasson IE, Sammel MD, Dokras A. Does intracytoplasmic sperm injection improve the fertilization rate and decrease the total fertilization failure rate in couples with well-defined unexplained infertility? A systematic review and meta-analysis. Fertil Steril 2013;100:704-11.

19. Kim MS, Kim J, Youm HW, Park JY, Choi HY, Jee BC. Embryonic development in human oocytes fertilized by split insemination. Obstet Gynecol Sci 2015;58:217-22.

20. Jaroudi K, Al-Hassan S, Al-Sufayan H, Al-Mayman H, Qeba M, Coskun S. Intracytoplasmic sperm injection and conventional in vitro fertilization are complementary techniques in management of unexplained infertility. J Assist Reprod Genet 2003;20: 377-81.

21. Hwang JL, Seow KM, Lin YH, Hsieh BC, Huang LW, Chen HJ, et al. IVF versus ICSI in sibling oocytes from patients with polycystic ovarian syndrome: a randomized controlled trial. Hum Reprod 2005;20:1261-5.
22. Yoeli R, Orvieto R, Ashkenazi J, Shelef M, Ben-Rafael Z, Bar-Hava I. Comparison of embryo quality between intracytoplasmic sperm injection and in vitro fertilization in sibling oocytes. J Assist Reprod Genet 2008;25:23-8.

23. Ming L, Yuan C, Ping Z, Ping L, Jie Q. Conventional in vitro fertilization maybe yields more available embryos than intracytoplasmic sperm injection for patients with no indications for ICSI. Int J Clin Exp Med 2015;8:21593-8.

24. Staessen C, Camus M, Clasen K, De Vos A, Van Steirteghem A. Conventional in-vitro fertilization versus intracytoplasmic sperm injection in sibling oocytes from couples with tubal infertility and normozoospermic semen. Hum Reprod 1999;14:2474-9.

25. Verheyen G, Tournaye H, Staessen C, De Vos A, Vandervorst M, Van Steirteghem A. Controlled comparison of conventional invitro fertilization and intracytoplasmic sperm injection in patients with asthenozoospermia. Hum Reprod 1999;14:2313-9.

26. Bukulmez O, Yarali H, Yucel A, Sari T, Gurgan T. Intracytoplasmic sperm injection versus in vitro fertilization for patients with a tubal factor as their sole cause of infertility: a prospective, randomized trial. Fertil Steril 2000;73:38-42.

27. Fishel S, Aslam I, Lisi F, Rinaldi L, Timson J, Jacobson M, et al. Should ICSI be the treatment of choice for all cases of in-vitro conception? Hum Reprod 2000;15:1278-83.

28. Taylor TH, Wright G, Jones-Colon S, Mitchell-Leef D, Kort HI, Nagy ZP. Comparison of ICSI and conventional IVF in patients with increased oocyte immaturity. Reprod Biomed Online 2008;17:4652.

29. Balakier H, Sojecki A, Motamedi G, Librach C. Time-dependent capability of human oocytes for activation and pronuclear formation during metaphase II arrest. Hum Reprod 2004;19:982-7.

30. Strassburger D, Friedler S, Raziel A, Kasterstein E, Schachter M, Ron-El R. The outcome of ICSI of immature MI oocytes and rescued in vitro matured MII oocytes. Hum Reprod 2004;19:158790.

31. Shu Y, Gebhardt J, Watt J, Lyon J, Dasig D, Behr B. Fertilization, embryo development, and clinical outcome of immature oocytes from stimulated intracytoplasmic sperm injection cycles. Fertil Steril 2007;87:1022-7.

32. Ko DS, Lee SH, Park DW, Yang KM, Lim CK. Pregnancy and fertilization potential of immature oocytes retrieved in intracytoplasmic sperm injection cycles. Clin Exp Reprod Med 2015;42:11825. 